\title{
An Asymptotic Formula for a Type of Singular Oscillatory Integrals
}

\author{
By L. C. Hsu and Y. S. Chou
}

\begin{abstract}
This paper offers a general expansion formula for oscillatory integrals of the form $\int_{0}^{1} x^{-a} f(x,\{N x\}) d x$ in which $N$ is a large parameter, $\{N x\}$ denotes the fractional part of $N x$, and $\alpha$ is a fixed real number in $0<\alpha<1$. Our formula is expressed in terms of some ordinary integrals with integrands containing periodic Bernoulli functions and the generalized Riemann zeta function.
\end{abstract}

1. Introduction. The object of this paper is to establish a general asymptotic formula for singular oscillatory integrals of the form

$$
I=\int_{0}^{1} x^{-\alpha} f(x,\{N x\}) d x \quad(0<\alpha<1),
$$

where $\{N x\}$ denotes the fractional part of $N x, N$ being a large real parameter. For the limiting case $\alpha \rightarrow 0+$ we have the expansion formula

$$
\begin{aligned}
\int_{0}^{1} f(x,\{N x\}) d x= & \int_{0}^{1} \int_{0}^{1} f(x, y) d x d y \\
& +\sum_{k=1}^{m-1} \frac{1}{k !}\left(\frac{1}{N}\right)^{k} \int_{0}^{1} H_{k}(y, N) d y+O\left(N^{-m}\right),
\end{aligned}
$$

where $f(x, y)$ is continuous on the square $[0,1] \times[0,1]$ with a continuous $m$ th partial derivative $f_{x}^{(m)}(x, y)=(\partial / \partial x)^{m} f(x, y)$, and $H_{k}(y, N)$ is defined by

$$
H_{k}(y, N)=f_{x}^{(k-1)}(1, y) \bar{B}_{k}(y-\{N\})-f_{x}^{(k-1)}(0, y) B_{k}(y),
$$

in which $B_{k}(x)$ and $\bar{B}_{k}(x)$ denote the Bernoulli polynomial of degree $k$ and the corresponding Bernoulli function with period unity, respectively, and $\{N\}$ may be written as $\{N\}=N-[N]$ with $[N]$ denoting the integral part of $N$.

The formula (2) was proved earlier and includes as special cases some useful asymptotic expansions offered by Erugin-Sobolev, Krylov, Riekstenš [5], and Havie [2], respectively. Detailed references may be found in our book [4]. As is known, one frequently encounters the particular cases that $f(x, y)$ is periodic with period 1 in $y$, and that the variables of $f(x, y)$ may be separated such as $f(x, y)=g(x)$. $h(y)$, etc. However, it may also happen in some practical problems that the variables of $f(x, y)$ cannot be separated.

Received October 1, 1980; revised February 4, 1981.

1980 Mathematics Subject Classification. Primary 41A60.

Key words and phrases. Periodic Bernoulli function, generalized Riemann zeta function, Euler summation formula. 
2. Statement of Result. In order to attain a general expansion for (1) we have to make use of the generalized zeta function $\zeta(s, a)$ defined, for $\operatorname{Re}(s)>1$, by the equation

$$
\zeta(s, a)=\sum_{n=0}^{\infty}(n+a)^{-s} \quad(a \neq 0,-1,-2, \ldots),
$$

and extended to the whole $s$-plane by analytic continuation except for a simple pole at $s=1$ with residue 1 . In particular, we shall employ the useful expansion

$$
\begin{aligned}
\zeta(s, a)= & \sum_{0<n<\omega}(n+a)^{-s}-\frac{(\omega+a)^{1-s}}{1-s} \\
& -\sum_{k=1}^{m-1} \frac{1}{k !}\left(\frac{1}{\omega+a}\right)^{s+k-1}(-1)^{k}\left[D^{k-1} x^{-s}\right]_{x=1} \bar{B}_{k}(\omega-) \\
& +O\left(\omega^{-m-s+1}\right),
\end{aligned}
$$

where $\operatorname{Re}(s)>1-m, s \neq 1, a>0$, and $D=d / d x$ is the differential operator. This expansion can be verified directly by a suitable application of the Euler summation formula with remainder for large real $\omega$, and by the aid of analytic continuation.

What we want to establish is the following

THEOREM. Let $f(x, y)$ be continuous on $R \equiv[0,1] \times[0,1]$ and have continuous partial derivatives, with respect to $x$, of orders up to $m+1$ on $R$. Then for $N$ large we have the asymptotic formula

$$
\begin{aligned}
\int_{0}^{1} x^{-\alpha} f(x, & \{N x\}) d x \\
= & \int_{0}^{1} \int_{0}^{1} x^{-\alpha} f(x, y) d x d y \\
& +\sum_{k=1}^{m-1} \frac{1}{k !}\left(\frac{1}{N}\right)^{k} \int_{0}^{1} F_{x}^{(k-1)}(1, y) \bar{B}_{k}(y-\{N\}) d y \\
& +\sum_{\mu=0}^{m-1} \frac{1}{\mu !}\left(\frac{1}{N}\right)^{-\alpha+\mu+1} \int_{0}^{1} f_{x}^{(\mu)}(0, y) \zeta(\alpha-\mu, y) d y+O\left(N^{-m}\right),
\end{aligned}
$$

where $0<\alpha<1, F(x, y)=x^{-\alpha} f(x, y)$ and $F_{x}^{(k-1)}(x, y)=(\partial / \partial x)^{k-1} F(x, y)$.

It is clear that one may write $f(x,\{N x\})=f(x, N x)$ in case $f(x, y)$ is periodic with period 1 in $y$, and that (2) may be regarded as the limiting case of (5) since

$$
\begin{gathered}
\lim _{\alpha \rightarrow 0+} \int_{0}^{1} x^{-\alpha} f(x,\{N x\}) d x=\int_{0}^{1} f(x,\{N x\}) d x, \\
\lim _{\alpha \rightarrow 0+} \zeta(\alpha-\mu, y)=\zeta(-\mu, y)=-B_{\mu+1}(y) /(\mu+1),
\end{gathered}
$$

where $\mu=0,1, \ldots, m-1 ; 0<y \leqslant 1$.

3. Proof of Theorem. This depends essentially upon a suitable application of (2) and involves manipulations of somewhat complicated expressions. First, utilizing both (4) and the symmetry relation for Bernoulli function $\bar{B}_{k}(x)=(-1)^{k} \bar{B}_{k}(1-x)$, 
and taking $s=\alpha-\mu$ and $\omega=N-a$, we have

$$
\begin{aligned}
\zeta(\alpha-\mu, a)= & \sum_{0<n<N-a}(n+a)^{\mu-\alpha}-\frac{N^{-\alpha+\mu+1}}{-\alpha+\mu+1} \\
& -\sum_{k=1}^{m-1} \frac{1}{k !}\left(\frac{1}{N}\right)^{\alpha+k-\mu-1}\left[D^{k-1} x^{-\alpha+\mu}\right]_{x=1} \cdot \bar{B}_{k}((a-\{N\})+) \\
& +O\left(N^{-m-\alpha+\mu+1}\right) .
\end{aligned}
$$

Let

$$
G(x, y)=x^{-\alpha} f(x, y)-\sum_{\mu=0}^{m} \frac{1}{\mu !} f_{x}^{(\mu)}(0, y) x^{\mu-\alpha}
$$

Then, by Taylor's expansion of $f(x, \cdot)$ with integral remainder, it is easy to verify that $G(x, y)$ possesses a continuous $m$ th partial derivative $G_{x}^{(m)}(x, y)$ with

$$
G_{x}^{(k-1)}(0, y)=\lim _{x \rightarrow 0} G_{x}^{(k-1)}(x, y)=0 \quad(k=1, \ldots, m)
$$

Now, applying formula (2) to the function $G(x, y)$ and taking account of (7), we obtain

(8)

$$
\begin{aligned}
\int_{0}^{1} G(x, & \{N x\}) d x \\
= & \int_{0}^{1} \int_{0}^{1} G(x, y) d x d y \\
& +\sum_{k=1}^{m-1} \frac{1}{k !}\left(\frac{1}{N}\right)^{k} \int_{0}^{1} G_{x}^{(k-1)}(1, y) \bar{B}_{k}(y-\{N\}) d y+O\left(N^{-m}\right) \\
= & J_{1}+J_{2}+O\left(N^{-m}\right), \text { say. }
\end{aligned}
$$

Here $J_{1}$ and $J_{2}$ as defined in (8) may be written as

$$
\begin{aligned}
J_{1}= & \int_{0}^{1} \int_{0}^{1} x^{-\alpha} f(x, y) d x d y-\sum_{\mu=0}^{m} \frac{1}{\mu !(1-\alpha+\mu)} \int_{0}^{1} f_{x}^{(\mu)}(0, y) d y, \\
J_{2}= & \sum_{k=1}^{m-1} \frac{1}{k !}\left(\frac{1}{N}\right)^{k} \int_{0}^{1} F_{x}^{(k-1)}(1, y) \bar{B}_{k}(y-\{N\}) d y \\
& -\sum_{\mu=0}^{m} \frac{1}{\mu !} \int_{0}^{1} f_{x}^{(\mu)}(0, y)\left\{\sum_{k=1}^{m-1} \frac{1}{k !}\left(\frac{1}{N}\right)^{k} \bar{B}_{k}(y-\{N\})\left[D^{k-1} x^{\mu-\alpha}\right]_{x-1}\right\} d y .
\end{aligned}
$$

Moreover, we have

$$
\begin{aligned}
\int_{0}^{1} G(x,\{N x\}) d x= & \int_{0}^{1} x^{-\alpha} f(x,\{N x\}) d x \\
& -\sum_{\mu=0}^{m} \frac{1}{\mu !} \int_{0}^{1} f_{x}^{(\mu)}(0,\{N x\}) x^{\mu-\alpha} d x
\end{aligned}
$$


and

$$
\begin{aligned}
\int_{0}^{1} f_{x}^{(\mu)}(0,\{N x\}) x^{\mu-\alpha} d x=\frac{1}{N} \int_{0}^{N} f_{x}^{(\mu)}(0,\{y\})\left(\frac{y}{N}\right)^{\mu-\alpha} d y \\
=\left(\frac{1}{N}\right)^{-\alpha+\mu+1}\left\{\int_{0}^{1} f_{x}^{(\mu)}(0, y) \sum_{0<i<[N]-1}(y+i)^{-\alpha+\mu} d y\right. \\
\left.\quad+\int_{0}^{\{N\}} f_{x}^{(\mu)}(0, y)(y+[N])^{-\alpha+\mu} d y\right\} \\
=\left(\frac{1}{N}\right)^{-\alpha+\mu+1} \int_{0}^{\{N\}} f_{x}^{(\mu)}(0, y) \sum_{0<i<N-y}(y+i)^{-\alpha+\mu} d y \\
+\left(\frac{1}{N}\right)^{-\alpha+\mu+1} \int_{\{N\}}^{1} f_{x}^{(\mu)}(0, y) \sum_{0<i<N-y}(y+i)^{-\alpha+\mu} d y \\
=\left(\frac{1}{N}\right)^{-\alpha+\mu+1} \int_{0}^{1} f_{x}^{(\mu)}(0, y) \sum_{0<i<N-y}(y+i)^{-\alpha+\mu} d y .
\end{aligned}
$$

Comparing (10) with (8) and noticing (9) and (11), we get

$$
\begin{aligned}
\int_{0}^{1} x^{-\alpha} f(x,\{N x\}) d x & \\
= & \int_{0}^{1} \int_{0}^{1} x^{-\alpha} f(x, y) d x d y \\
& +\sum_{k=1}^{m-1} \frac{1}{k !}\left(\frac{1}{N}\right)^{k} \int_{0}^{1} F_{x}^{(k-1)}(1, y) \bar{B}_{k}(y-\{N\}) d y+\sum_{\mu=0}^{m} \frac{1}{\mu !}\left(\frac{1}{N}\right)^{-\alpha+\mu+1} \\
& \quad \int_{0}^{1} f_{x}^{(\mu)}(0, y)\left\{\sum_{0<i<N-y}(y+i)^{-\alpha+\mu}-\frac{N-\alpha+\mu+1}{1-\alpha+\mu}\right. \\
& +O\left(N^{-m}\right) .
\end{aligned}
$$

Notice that the expression involved in the braces $\{\cdots\}$ of the right-hand side of (12) is equivalent to $\zeta(\alpha-\mu, y)+O\left(N^{-m-\alpha+\mu+1}\right)$ in accordance with (6). Hence the theorem is proved.

4. Remarks. The asymptotic formula (5) can be specialized in various ways. In particular, for the case $\alpha \rightarrow 0+$ and $N$ being a large integer parameter, it reduces to a much simpler expression that has certain applications to numerical integration methods (cf. [3] and [4]). As for the general case $0<\alpha<1$, the generalized zeta function $\zeta(\alpha-\mu, y)(\mu=1,2, \ldots)$ involved in (5) may sometimes be treated more conveniently by using the Hurwitz series representation (with $\operatorname{Re}(s)<0$, $0<a<1)$

$$
\zeta(s, a)=\frac{2 \Gamma(1-s)}{(2 \pi)^{1-s}} \sum_{n=1}^{\infty} n^{s-1} \sin \left(2 n \pi a+\frac{\pi s}{2}\right)
$$


instead of the asymptotic expression (6). Thus it may be observed that the expansion formula for the familiar integral

$$
J=\int_{\alpha}^{\beta}(t-\alpha)^{\lambda-1} \varphi(t) e^{i N t} d t \quad(0<\lambda<1, i=\sqrt{-1}),
$$

as stated in Davis-Rabinowitz [1, p. 120], is also implied by (5) as a special case.

Department of Mathematics

Dalian Institute of Technology

Lüda, Liaoning, People's Republic of China

Department of Mathematics

Jilin University, Changchun

People's Republic of China

1. P. J. DAvis \& P. RABINowItZ, Methods of Numerical Integration, Blaisdell, Waltham, Mass., 1975, p. 120.

2. T. HAVIE, "Remarks on an expansion for integrals of rapidly oscillating functions," $B I T$, v. 13 , 1973, pp. 16-29.

3. L. C. Hsu, "A refinement of the line integral approximation method and its application," Sci. Record (N. S. Academia Sinica), No. 6, 1958, pp. 193-196.

4. L. C. Hsu \& Y. S. CHOU, Numerical Integration in Higher Dimensions, Science Press, Peking, 1980, Chapter 14. (Chinese)

5. E. RIEKSTENš, "On asymptotic expansions of some integrals involving a large parameter," Učen. Zap. Leningrad. Gos. Univ., v. 41, 1961, pp. 5-23. (Russian) 